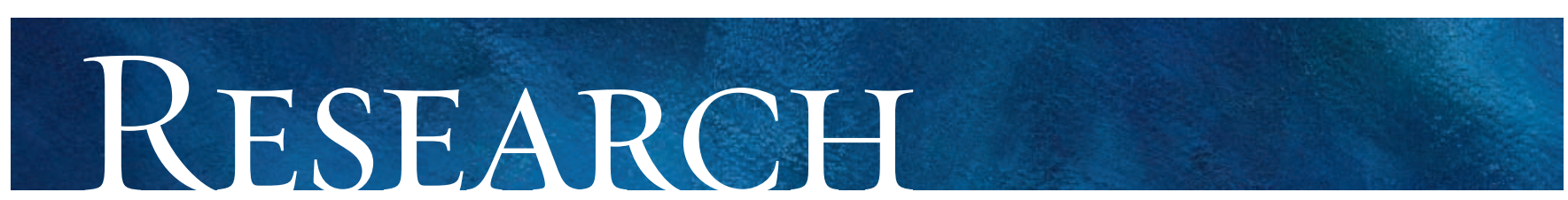

\title{
Effects of exercise on breast cancer patients and survivors: a systematic review and meta-analysis
}

\author{
Margaret L. McNeely, Kristin L. Campbell, Brian H. Rowe, Terry P. Klassen, John R. Mackey, \\ Kerry S. Courneya
}

\section{ABSTRACT}

Background: Physical exercise has been identified as a potential intervention to improve quality of life in women with breast cancer. We sought to summarize the available evidence concerning the effects of exercise on breast cancer patients and survivors.

Methods: We searched the Cochrane Central Register of Controlled Trials, MEDLINE, EMBASE, CINAHL, PsychINFO, CancerLit, PEDro and SportDiscus as well as conference proceedings, clinical practice guidelines and other unpublished literature resources. We included only randomized controlled trials that examined exercise interventions for breast cancer patients or survivors with quality of life, cardiorespiratory fitness or physical functioning as primary outcomes. We also extracted data on symptoms of fatigue, body composition and adverse effects.

Results: Of 136 studies identified, 14 met all the inclusion criteria. Despite significant heterogeneity and relatively small samples, the point estimates in terms of the benefits of exercise for all outcomes were positive even when statistical significance was not achieved. Exercise led to statistically significant improvements in quality of life as assessed by the Functional Assessment of Cancer Therapy-General (weighted mean difference [WMD] 4.58, 95\% confidence interval [CI] 0.35 to 8.80) and Functional Assessment of Cancer Therapy-Breast (WMD 6.62, 95\% Cl 1.21 to 12.03). Exercise also led to significant improvements in physical functioning and peak oxygen consumption and in reducing symptoms of fatigue.

Interpretation: Exercise is an effective intervention to improve quality of life, cardiorespiratory fitness, physical functioning and fatigue in breast cancer patients and survivors. Larger trials that have a greater focus on study quality and adverse effects and that examine the long-term benefits of exercise are needed for this patient group.

CMAJ 2006;175(I):34-4I

A mong women living in Western countries, breast cancer is an important disease in terms of incidence and mortality. ${ }^{1-3}$ Improvement in survival over the past 20 years has resulted in a substantial number of breast cancer survivors, many of whom will have a normal life ex- pectancy. ${ }^{3}$ Increasingly, cancer care is being directed toward developing interventions to improve overall quality of life as well as longevity. ${ }^{4}$

Physical exercise has consistently been identified as a central element of rehabilitation for many chronic diseases ${ }^{5-8}$ and has been successful in improving quality of life and reducing all-cause mortality. ${ }^{9}$ Recent observational evidence suggests that moderate levels of physical activity may even reduce the risk of death from breast cancer, ${ }^{10}$ and therefore exercise may prove to be a valuable intervention to improve not only quality of life but overall survival.

The effectiveness of exercise interventions in cancer patients and survivors has been assessed in both qualitative systematic reviews and meta-analyses that included all types of cancers and all types of trial designs (i.e., nonrandomized, uncontrolled trials). ${ }^{11-13}$ It is well known, however, that cancer survivor groups are clinically heterogeneous in terms of their demographic profile (e.g., age, sex distribution), behavioural profile (e.g., smoking status, alcohol consumption, obesity), disease pathophysiology, treatment protocols, and symptoms and side effects. Consequently, the wisdom of summarizing the effects of exercise interventions across such disparate groups is questionable. It is clear from previous reviews that the vast majority of exercise intervention research has involved breast cancer patients and survivors. In addition, there are now newer studies, so there is sufficient research available to restrict a meta-analysis to this cancer survivor group. It is also well known that the inclusion of nonrandomized or uncontrolled trials leads to an overestimation of the effect of an intervention. It is recommended that meta-analyses be restricted to randomized controlled trials (RCTs) whenever possible. ${ }^{14}$ Here, we present a systematic quantitative review of RCTs on the effects of exercise interventions on breast cancer patients and survivors.

\section{Methods}

We searched the following electronic databases to March 2005: Cochrane Central Register of Controlled Trials, MEDLINE, EMBASE, CancerLit, CINAHL, PsychINFO, PEDro and SportDiscus. The breast cancer specialized register maintained by the Cochrane Breast Cancer Group was also searched. We used search terms related to breast cancer (e.g., breast neoplasms, mastectomy, axillary dissection), exercise 
(e.g., exercise, physical activity, sport) and publication type (e.g., random allocation, clinical trial). This search strategy was modified as necessary for each database; appropriate non-English language publications were not found. To locate unpublished research, we reviewed proceedings from major cancer and sports medicine meetings as well as clinical practice guidelines for breast cancer, and we searched Web sites housing clinical trial details, theses or dissertations. In addition, we hand-searched the reference lists of all potentially relevant studies and contacted experts and authors of previous studies to identify relevant articles.

Studies were considered eligible for inclusion if they were RCTs comparing exercise with a placebo, controlled comparison or standard care. For the purposes of the review, exercise was defined as a form of leisure-time physical activity that was performed on a repeated basis over an extended period of time, with the intention of improving fitness, performance or health. ${ }^{15}$ Studies with an additional treatment arm or combined intervention (e.g., exercise with diet modification) were included only if the effects of exercise could be isolated. Exercise studies that included cancers other than breast cancer were excluded unless separate data were available for the breast cancer subgroup. Therapeutic exercise regimens addressing only specific impairments related to the shoulder, arm or both were not included. A priori, we excluded reports that were available only in abstract form.

Trials were included only if they involved women with early to later stage (Stage O-III) breast cancer or who had undergone breast cancer surgery with or without adjuvant cancer therapy. Studies were required to have as a primary outcome quality of life, cardiorespiratory fitness or physical functioning. Secondary outcomes of interest included symptoms of fatigue and body composition (body weight or body mass index [BMI]). We also extracted data on adverse events resulting from the exercise intervention.

Two independent reviewers (MLM, KLC) screened the titles and abstracts of identified studies for eligibility. Papers deemed potentially relevant were obtained, and the full papers were reviewed for inclusion by the same 2 independent reviewers. Information on patients, methods, interventions, outcomes and adverse events were extracted from the original reports by the 2 independent reviewers onto paper forms that they had designed and pre-tested. Disagreements were resolved by consensus (MLM, KLC, KSC). The methodologic quality of each RCT was assessed using the following criteria:

I) Was there adequate concealment of allocation?

2) Was the method of randomization well described and appropriate?

3) Was the outcome assessment described as blinded?

4) Was the method of blinding of the assessment of outcomes well described and appropriate?

5) Was there a description of withdrawals and drop-outs?

6) Was the analysis intention-to-treat?

7) Were withdrawals and drop-outs less than Io\%?

8) Was adherence to the exercise intervention (attendance or completion of exercise session) greater than $70 \%$ ?

All items were scored as positive (+), negative (-) or un- clear (?). Studies were defined as being of "high quality" if they fulfilled 4 or more of the 8 quality criteria.

Study results were pooled, if appropriate, using random effects models after heterogeneity among the trials was considered. For continuous outcomes, individual study mean differences were reported; pooled statistics were calculated using weighted mean differences (WMD) when data were on a uniform scale and using standardized mean differences (SMD) when data were on different scales. All results were calculated with $95 \%$ confidence intervals (CIs). The estimated effect size was calculated for outcomes that were reported in 3 or more studies. For dichotomous variables, individual and pooled statistics were calculated as odds ratios (ORs) with $95 \%$ CIs. Heterogeneity was tested using a $\chi^{2}$ test that considered a $p$ value of less than 0.10 to indicate significant heterogeneity. When heterogeneity was evident and could be explained by clinical dissimilarities, trials were not pooled.

\section{Results}

We identified I40 papers, of which 25 were considered potentially relevant. ${ }^{16-40}$ Independent review of these 25 papers led to the inclusion of I4 studies involving 7I7 participants. ${ }^{16-20,25,26,30-32,34-37}$ Study methodology varied significantly, particularly with regards to timing of the exercise intervention, the chosen exercise regimen and outcomes reported (Table I). Kappa statistics for agreement between the reviewers on inclusion of trials and quality score were 0.8 and 0.92 respectively.

The median score for methodologic quality of all included studies was 3, with a range of o-8 (Table 2). Using a cutoff point of 4 out of 8 criteria, 4 of the I4 studies were considered high quality. ${ }^{18,19,30,37}$ The most common methodologic shortcomings in the included studies were failure to blind the outcome assessment (I2 studies scored "negative" or "unclear"); inadequate method of blinding outcome assessment (I2 studies scored "negative" or "unclear"); and inadequate concealment of allocation (II studies scored "negative" or "unclear").

Three studies involving 194 patients compared exercise with usual care. ${ }^{17,18,37}$ Exercise was superior to usual care for both the Functional Assessment of Cancer Therapy-General (FACT-G) and Functional Assessment of Cancer TherapyBreast (FACT-B) quality-of-life scales. Pooled data from these 3 studies demonstrated that exercise led to significant improvements in quality of life using both the FACT-G (WMD $4.58,95 \% \mathrm{CI} 0.35$ to 8.8 ) and FACT-B (WMD 6.62, 95\% CI I.2I to I2.03) scales (Fig. I).

Cardiorespiratory fitness was reported as an outcome in 9 studies $^{17-20,25,31,32,34,37}$ involving 473 patients. Owing to significant heterogeneity between the 9 trials, data were not combined and are reported only by specific outcome measurement (Table 3). Three of the studies ${ }^{18-20}$ that reported peak oxygen consumption in $\mathrm{mL} / \mathrm{kg}$ per minute from symptomlimited graded exercise tests were successfully combined. The pooled results from the 3 studies demonstrated a significant improvement in peak oxygen consumption with exercise (WMD 3.39, 95\% CI I.67 to 5.I0). 
Four studies ${ }^{18,20,35,37}$ monitored body weight, and 4 studies $^{18,20,34,35}$ reported BMI as an outcome. The pooled results from the 4 studies monitoring body weight showed a nonsignificant reduction (WMD $-0.03 \mathrm{~kg}, 95 \% \mathrm{CI}-0.44$ to 0.38 ). The individual study results, as well as the pooled results, for BMI also showed nonsignificant reductions in favour of exercise (WMD -0.02, 95\% CI -0.09 to o.05) (Table 3).

Four studies ${ }^{17,18,26,37}$ involving 208 patients reported physical functioning or physical well-being components of quality of life. Two ${ }^{17,18}$ used the physical well-being subscale of the FACT quality-of-life scale, and the other $2^{26,37}$ used the physical functioning subscale of the Medical Outcomes Trust 36-item Short Form Survey. ${ }^{41}$ The pooled results of all 4 studies showed a statistically significant increase in physical functioning and well-being from exercise (SMD 0.84, 95\% CI 0.36 to I.32) (Table 3).

Six studies ${ }^{16-18,20,30,34}$ involving 3 I9 patients assessed the effect of exercise on symptoms of fatigue. One study ${ }^{18}$ measured fatigue using the Functional Assessment of Cancer Therapy-Fatigue (FACT-F) quality-of-life scale, 4 studies $^{16,17,20,30}$ used the revised Piper Fatigue Scale and one study used a visual analogue scale for fatigue. ${ }^{34}$ Although all of the studies showed improvements in symptoms of fatigue with exercise, only $2^{18,34}$ reported statistically significant improvements. These 2 studies ${ }^{18,34}$ were also the only studies carried out following cancer treatment. The pooled results from all 6

Table 1: Characteristics of randomized controlled trials examining the effectiveness of exercise for breast cancer

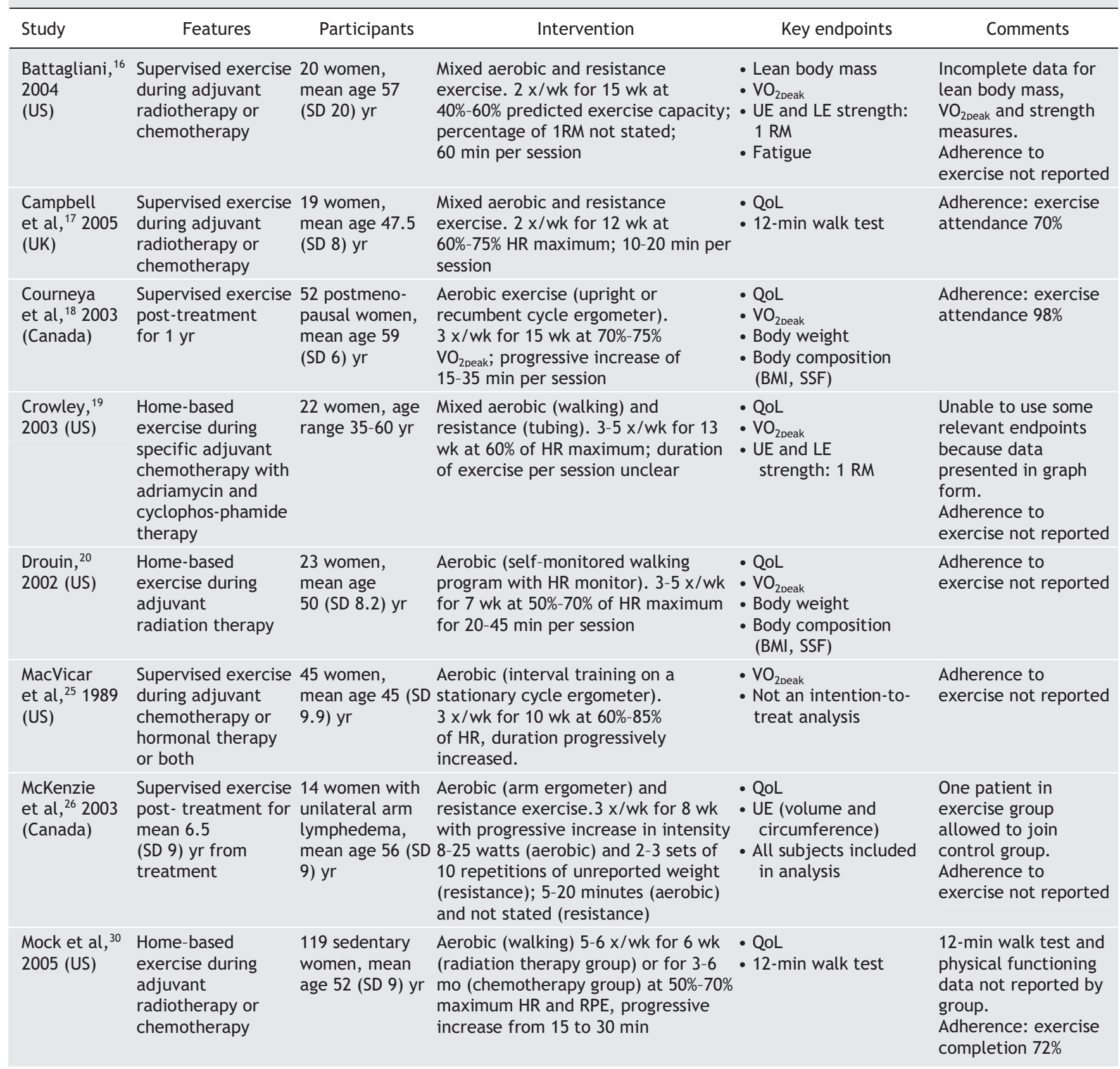


studies (Fig. 2) showed that exercise significantly improved symptoms of fatigue (SMD $0.46,95 \%$ CI 0.23 to 0.70 ). The pooled results from the 4 studies ${ }^{16,17,20,30}$ carried out during adjuvant cancer treatment showed a nonsignificant effect on fatigue (SMD 0.28, 95\% CI -0.02 to 0.57).

Adverse events from exercise programs were reported in 4 studies. ${ }^{18-20,36}$ There were reports of back injury $(n=4)$ and shoulder tendinitis $(n=\mathrm{I})$ related to participation in the resistance exercise intervention during the first 6 months of one trial. ${ }^{36}$ Injuries to the back $(n=4)$, wrist $(n=1)$, lower leg and ankle $(n=5)$ and rotator cuff $(n=\mathrm{I})$ related to study participation were also reported in months $7-\mathrm{I} 2$ of the same trial. In another study, shoulder tendinitis $(n=\mathrm{I})$ and a wors- ening of fatigue $(n=2)$ were reported as adverse outcomes related to study participation. ${ }^{20}$ Cases of lymphedema occurring in exercise participants were reported in 2 studies. ${ }^{18,19}$ There was a nonsignificant difference in the occurrence of lymphedema between exercise and control interventions in the individual studies and when data were pooled (OR 4.9I, $95 \%$ CI 0.52 to 36.25 ).

\section{Interpretation}

This review summarizes the best available evidence regarding the effects of exercise on quality of life and physical outcomes for breast cancer patients and survivors.

Table 1: Characteristics of randomized controlled trials examining the effectiveness of exercise for breast cancer (continued)

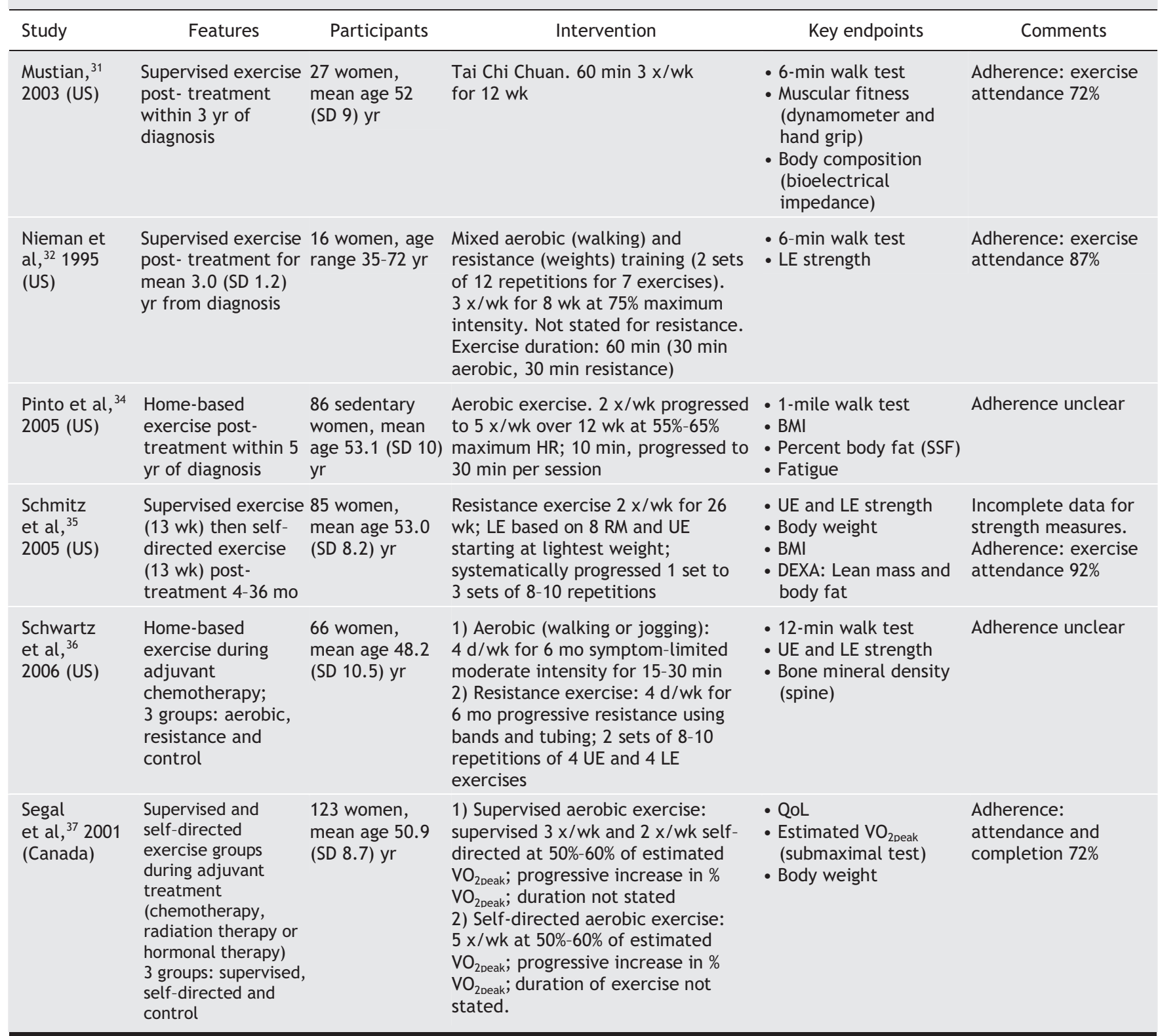

Note: $\mathrm{SD}=$ standard deviation, $\mathrm{HR}=$ heart rate, $\mathrm{QoL}=$ quality of life, $\mathrm{VO}_{2 \text { peak }}=$ peak oxygen consumption, measured using an incremental exercise test (aerobic fitness), $\mathrm{BMI}=$ body mass index, SSF = sum of skin folds, UE = upper extremity, LE = lower extremity, RM = repetition maximum for muscular strength, DEXA = dual-energy $\mathrm{x}$-ray absorptiometry, RPE = rating of perceived exertion. 
Table 2: Methodologic quality assessment of randomized controlled trials of the effectiveness of exercise interventions for breast cancer

\begin{tabular}{|c|c|c|c|c|c|c|c|c|c|}
\hline \multirow[b]{2}{*}{ Study } & \multicolumn{8}{|c|}{ Criteria* } & \multirow[b]{2}{*}{ Total/8 } \\
\hline & 1 & 2 & 3 & 4 & 5 & 6 & 7 & 8 & \\
\hline Battagliani, ${ }^{16} 2004$ & $?$ & + & - & - & $?$ & + & + & - & 3 \\
\hline Campbell et al, ${ }^{17} 2005$ & $?$ & + & - & - & + & - & - & + & 3 \\
\hline Courneya et al, ${ }^{18} 2003$ & + & + & + & + & + & - & + & + & 7 \\
\hline Crowley, ${ }^{19} 2003$ & + & + & + & + & + & - & + & $?$ & 6 \\
\hline Drouin, ${ }^{20} 2002$ & $?$ & + & - & - & $?$ & - & + & $?$ & 2 \\
\hline MacVicar et al, ${ }^{25} 1989$ & $?$ & - & $?$ & $?$ & - & - & - & $?$ & 0 \\
\hline McKenzie et al, ${ }^{26} 2003$ & $?$ & - & - & - & + & + & + & $?$ & 3 \\
\hline Mock et al, ${ }^{30} 2005$ & + & + & - & - & + & + & - & + & 5 \\
\hline Mustian, ${ }^{31} 2003$ & $?$ & - & $?$ & $?$ & + & - & - & + & 2 \\
\hline Nieman et al, 321995 & $?$ & - & - & - & + & - & - & + & 2 \\
\hline Pinto et al, ${ }^{34} 2005$ & $?$ & - & - & - & + & + & + & $?$ & 3 \\
\hline Schmitz et al, ${ }^{35} 2005$ & $?$ & + & - & - & + & - & - & + & 3 \\
\hline Schwartz et al, ${ }^{36} 2006$ & - & - & - & - & - & + & + & $?$ & 2 \\
\hline Segal et al, ${ }^{37} 2001$ & $?$ & + & - & - & + & + & - & + & 4 \\
\hline No. of studies meeting criterion & 3 & 8 & 2 & 2 & 10 & 6 & 7 & 7 & - \\
\hline
\end{tabular}

Note: + = positive, - = negative, $?=$ unclear.

*1) Adequate allocation concealment, 2) adequate method of randomization, 3) blinded outcome assessment, 4) adequate method of blinding, 5) description of withdrawals or drop-outs, 6) intention-to-treat analysis, 7) withdrawals and drop-outs $<10 \%, 8$ ) adherence (reported attendance or completion of exercise sessions) $>70 \%$.

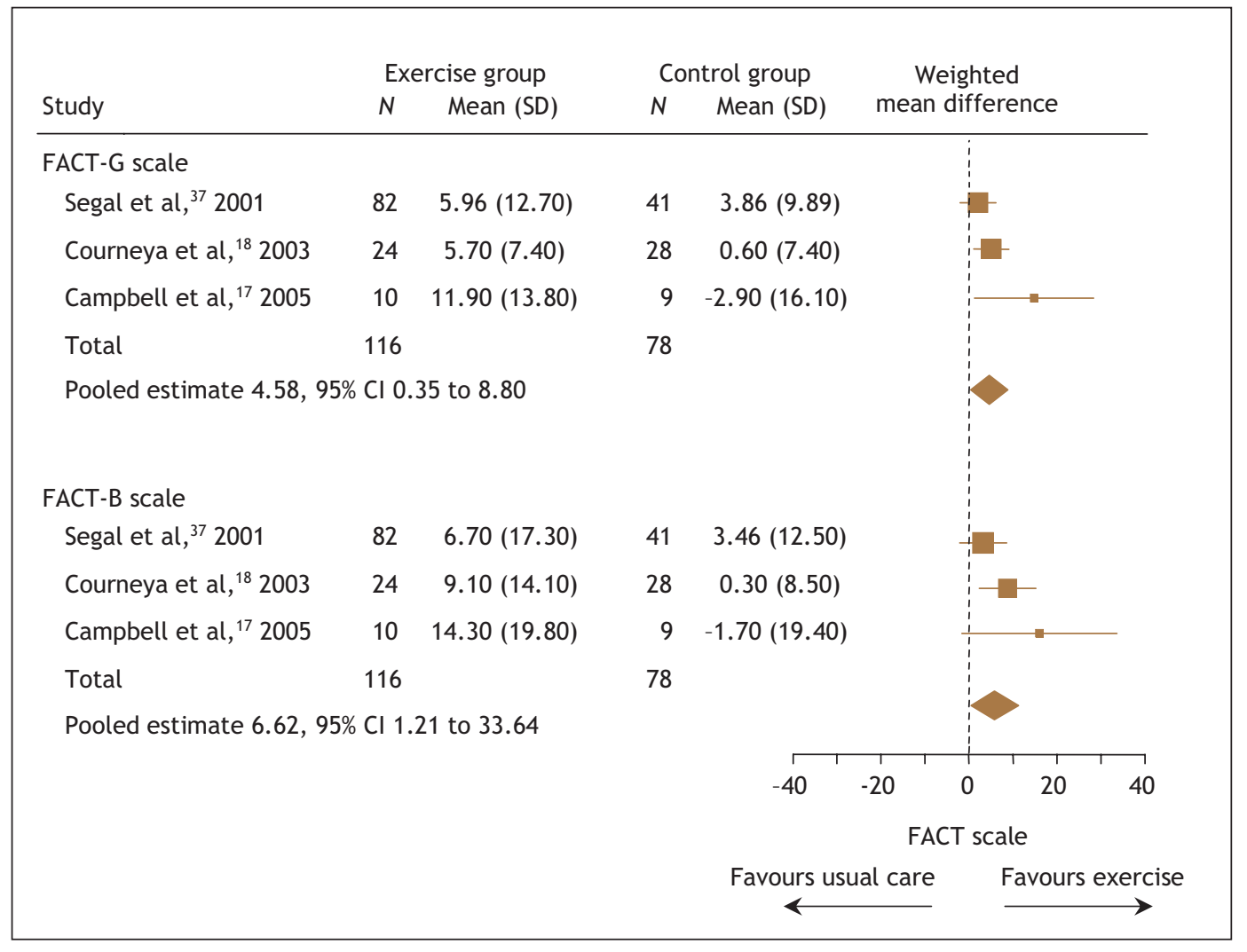

Fig. 1: Pooled effects of exercise on quality of life from clinical trials involving breast cancer patients. FACT-G = Functional Assessment of Cancer Therapy-General, FACT-B = Functional Assessment of Cancer Therapy-Breast. 
Only 3 studies provided adequate data to assess quality of life. The pooled estimate showed that a statistically significant increase of greater than 4.0 points on the FACT scale represents a clinically meaningful improvement in quality of life from exercise. ${ }^{42}$ Additionally, analyses of the physical functioning and physical well-being subscales of quality of life indicated large improvements (effect size $=0.84$ ) from exercise.

The pooled results of 3 studies examining peak oxygen consumption from symptom-limited graded exercise testing showed an improvement of $3.39 \mathrm{~mL} / \mathrm{kg}$ per minute or almost one metabolic equivalent (MET) improvement in fitness. ${ }^{43}$ Each I MET increment in fitness has been found to correspond to a $12 \%$ improvement in survival in men. ${ }^{43}$ Since cardiorespiratory fitness is an important predictor of all-cause mortality in women, ${ }^{9}$ it is possible that an improvement of this magnitude would have similar implications in women; however, the duration of these studies was insufficient to provide firm evidence.

The pooled results of the 6 studies examining the effect of exercise on symptoms of fatigue showed a moderate-to-large effect (effect size $=0.72$ ); however, statistically significant improvements in symptoms of fatigue were reported in only 2 studies. ${ }^{18,34}$ Both studies examined exercise following primary cancer treatment. ${ }^{18,34}$ During adjuvant cancer treatment, no effect of exercise on fatigue was found. The evidence suggests that exercise has a nonsignificant and potentially small effect on symptoms of fatigue for women undergoing adjuvant cancer treatment. Despite statistical nonsignificance in the 4 studies, all point estimates were in favour of exercise, which suggests the need for more research before rejecting this effect.

There was no statistically or clinically significant change in body weight or BMI as a result of the exercise trials included in this review. It is not known, however, whether positive changes in body composition occurred as a result of the exercise intervention because there was a lack of studies using direct measures of tissue and body composition. As an example, Schmitz and associates examined body composition by means of dual x-ray absorptiometry and reported positive changes in lean body mass as well as significant decreases in percent body fat in favour of the exercise intervention (Table 3). ${ }^{35}$ As well, Schwartz and colleagues assessed bone density of the lumbar spine using dual x-ray absorptiometry and reported that subjects participating in weight-bearing aerobic exercise had significantly less bone density loss than control subjects (Table 3 ). ${ }^{36}$ This suggests that positive changes in body composition may occur despite nonsignificant changes in body weight and BMI.

The I4 studies included in this review were of variable quality, and only 4 were considered to be of high quality. Our conclusions are tempered by this fact. Clearly, further progress must be made to improve research quality. Future trials should focus on adequate randomization, concealment of allocation and blinding of outcome assessors throughout the study.

A noteworthy feature of trials included in this review was the wide variability in study interventions. Many different exercise regimens were prescribed. The diversity in exercise prescription is not surprising, given the lack of consensus on the optimal exercise prescription for this patient population. Conversely, the wide variety in study outcomes and measurement methods is surprising. This variation precluded pooling studies and made overall conclusions regarding the relative effectiveness of exercise difficult. The short duration or complete lack of follow-up data examining the effect of exercise on quality of life and rehabilitative outcomes in the long term is also noted. Moreover, data are lacking to support the use of exercise in preventing cancer recurrence and improving overall survival.

Table 3: Effects of exercise on cardiorespiratory fitness, body composition and physical functioning

\begin{tabular}{|c|c|c|c|c|c|c|}
\hline Outcome & $\begin{array}{l}\text { No. of } \\
\text { studies }\end{array}$ & $N$ & $\begin{array}{l}\text { Weighted mean } \\
\text { difference }(95 \% \mathrm{Cl})\end{array}$ & $p$ value & $\begin{array}{l}\text { Standardized mean difference } \\
\text { (effect size) }(95 \% \mathrm{Cl})\end{array}$ & $p$ value \\
\hline \multicolumn{7}{|l|}{ Cardiorespiratory fitness } \\
\hline $\mathrm{VO}_{2 \text { peak }}$ relative, $\mathrm{mL} / \mathrm{kg}$ per $\min$ & 3 & 95 & $3.39(1.67$ to 5.1$)$ & $0.0001^{*}$ & $1.14(0.47$ to 1.81$)$ & $0.0009^{*}$ \\
\hline Predicted $\mathrm{VO}_{2}, \mathrm{~mL} / \mathrm{kg}$ per min & 2 & 150 & $0.99(-0.21$ to 2.18$)$ & 0.07 & Not estimated & - \\
\hline 1-mile walk test, min & 1 & 89 & $-1.31(-0.42$ to -0.20$)$ & $0.004^{*}$ & Not estimated & - \\
\hline \multicolumn{7}{|l|}{ Body composition } \\
\hline Weight, kg & 4 & 277 & $-0.03(-0.44$ to 0.38$)$ & 0.88 & $-0.07(-0.36$ to 0.21$)$ & 0.61 \\
\hline Body mass index, $\mathrm{kg} / \mathrm{m}^{2}$ & 4 & 240 & $-0.02(-0.09$ to 0.05$)$ & 0.58 & $-0.12(-0.38$ to 0.13$)$ & 0.35 \\
\hline Physical functioning & 4 & 208 & Not estimated & - & $0.84(0.36$ to 1.32$)$ & 0.0006 \\
\hline
\end{tabular}

Note: $\mathrm{Cl}=$ confidence interval.

*Indicates significant value. 


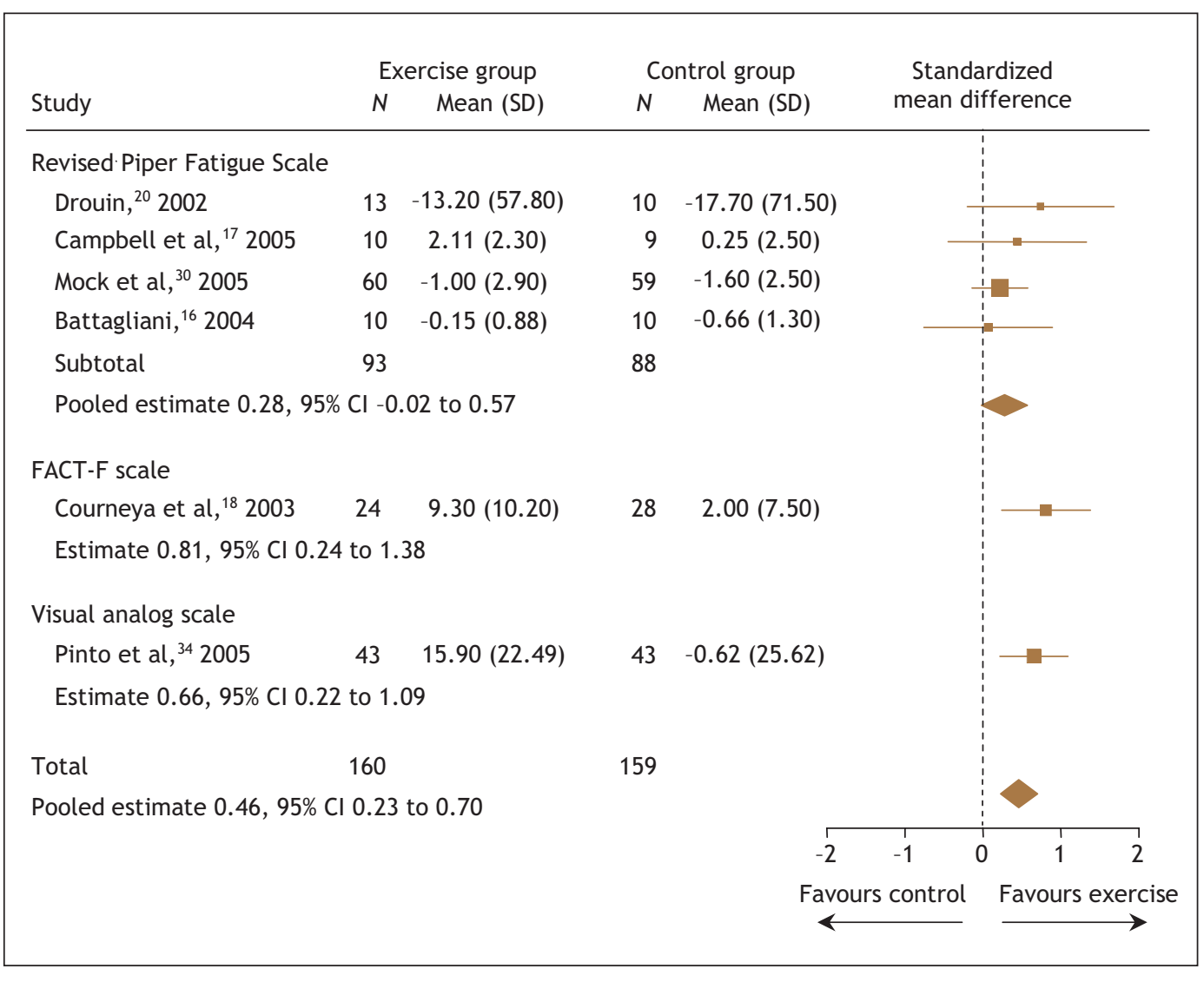

Fig. 2: Pooled effects of exercise on symptoms of fatigue from clinical trials involving breast cancer patients. FACT-F = Functional Assessment of Cancer Therapy-Fatigue.

A further limitation is the nonspecificity with respect to the timing of the exercise intervention. Clinical heterogeneity was evident, particularly in trials carried out during adjuvant cancer treatment. This resulted from trials in which the participants were undergoing one of a variety of adjuvant treatments (e.g., chemotherapy, radiation therapy and hormonal therapy).

Finally, poor adverse event reporting in most of the studies limits any conclusions about the relative safety of exercise, and the small samples provide insufficient power to detect meaningful differences in rates of rare adverse events. For example, lymphedema is a potential side effect of cancer treatment and represents a barrier to exercise for some patients, ${ }^{4}$ yet none of the included studies formally monitored for this side effect.

The evidence suggests that exercise is an effective intervention to improve quality of life, cardiorespiratory fitness, physical functioning and symptoms of fatigue in breast cancer patients and survivors. Although these preliminary results are promising, the findings are based on a relatively small number of trials with significant methodologic weaknesses. Furthermore, there is currently no evidence to support the use of exercise regimens to reduce body weight or BMI. On the basis of our findings, we make the following research recommendations:

I. Methodologically rigorous studies designed to examine different exercise regimens (e.g., moderate v. low-intensity) are needed to better understand the role of physical exercise among breast cancer patients and survivors.

2. The exercise prescription should be reported in detail (frequency, intensity, time and type of exercise) to allow for determination of exercise dose-response. To this end, adherence to exercise should be reported for both completion of exercise sessions (attendance) and exercise prescription (intensity and duration). Furthermore, monitoring of activity in the comparison group(s) is necessary to assess potential contamination.

3. Consensus is required on standardized methods of assessing physical fitness and body composition to allow for pooling of data and for comparisons across studies.

4. Future trials should formally monitor for, and report the incidence of, potential adverse events such as lymphedema.

This article has been peer reviewed.

From the Department of Behavioural Medicine, Faculty of Physical Education, University of Alberta (McNeely, Campbell, Courneya), the Department of Emergency Medicine, University of Alberta and Capital Health (Rowe), the Department of Paediatrics, Stollery Children's Hospital and University of Alberta (Klassen) and the Department of Medical Oncology, Cross Cancer Institute and University of Alberta (Mackey), Edmonton, Alta.

Competing interests: None declared for Margaret McNeely, Kristin Campbell, Brian Rowe or Terry Klassen. Kerry Courneya and John Mackey were investigators in one of the primary studies (reference 8 ). 
Contributors: Margaret McNeely conceived the project, developed the proto$\mathrm{col}$, conducted searches, coordinated reviewers, data collection and extraction, and drafted the manuscript. Kristin Campbell conceived the project and contributed substantially to the protocol, article selection, data collection and extraction, and to the manuscript preparation. Brian Rowe contributed methodologic expertise as well as to developing the protocol and to the manuscript preparation. Terry Klassen contributed methodologic expertise as well as to developing the protocol. John Mackey contributed clinical expertise as well as to developing the protocol. Kerry Courneya contributed substantially to the content as well as to the protocol, article selection and manuscript preparation. All of the authors revised the article critically for content and gave final approval of the version to be published.

Acknowledgements: Margaret McNeely and Kristin Campbell are supported by full-time Health Research Studentships from the Alberta Heritage Foundation for Medical Research. Brian Rowe and Kerry Courneya are supported by the Canada Research Chairs Program; Kerry Courneya is also supported by a Research Team Grant from the National Cancer Institute of Canada with funds from the Canadian Cancer Society and the Sociobehavioral Cancer Research Network.

We thank Ms. Natasha Wiebe for her assistance with data entry and analysis and Ms. Sandra Blitz for her assistance with the figures.

\section{REFERENCES}

I. American Cancer Society. Cancer facts and figures 2004. Available: www.cancer.org/docroot/STT/stt_o_2004.asp?sitearea=STT\&level=I (accessed 2006 May 8).

2. Canadian Cancer Society. Canadian Cancer Statistics 2004. Available: www.cancer.ca /ccs/internet/mediareleaselist/o,,3172_21050487I_I9463455I_langId-en,oo.html (accessed 2006 May 8).

3. Jassem J, Buchanan M, Janicke F, et al. The Hamburg statement: the partnership driving the European agenda on breast cancer. Eur J Cancer 2004;40:1810-I.

4. Brown JK, Byers T, Doyle C, et al. Nutrition and physical activity during and after cancer treatment: an American Cancer Society guide for informed choices. $C A$ Cancer J Clin 2003;53:268-91.

5. Nixon S, O'Brien K, Glazier RH, et al. Aerobic exercise interventions for adults living with HIV/AIDS. In: The Cochrane Library; Issue 2, 2002. Oxford: Update Software.

6. Schulz KH, Gold SM, Witte J, et al. Impact of aerobic training on immune-endocrine parameters, neurotrophic factors, quality of life and coordinative function in multiple sclerosis. J Neurol Sci 2004;225:II-8.

7. Atlantis E, Chow CM, Kirby A, et al. An effective exercise-based intervention fo improving mental health and quality of life measures: a randomized controlled trial. Prev Med 2004;39:424-34

8. Taylor RS, Brown A, Ebrahim S, et al. Exercise-based rehabilitation for patient with coronary heart disease: systematic review and meta-analysis of randomized controlled trials. Am JMed 2004; II6:682-92.

9. Farrell SW, Braun L, Barlow CE, et al. The relation of body mass index, cardiorespiratory fitness, and all-cause mortality in women. Obes Res 2002;10:417-23.

ro. Holmes MD, Chen WY, Feskanich D, et al. Physical activity and survival after breast cancer diagnosis. JAMA 2005;293:2479-86.

II. Galvao DA, Newton RU. Review of exercise intervention studies in cancer patients. JClin Oncol 2005;23:899-909.

I2. Knols R, Aaronson NK, Duebelhart D, et al. Physical exercise in cancer patient during and after medical treatment: a systematic review of randomized and controlled trials. JClin Oncol 2005;23:3830-4I.

I3. Stevinson C, Lawlor DA, Fox KR. Exercise interventions for cancer patients: systematic review of controlled trials. Cancer Causes Control 2004;15:1035-56.

14. Altman DG, Schulz KF, Moher D, et al. The Revised CONSORT statement for reporting randomized trials: explanation and elaboration. Ann Intern Med 2001;134:663-94

I5. Bouchard C, Shephard RJ. Physical activity, fitness, and health: The model and key concepts. In: Bouchard CS, Shephard RJ, Stephens T, editors. Physical activity, fit ness, and health: international proceedings and consensus statement. Champaign, IL: Human Kinetics; 1994. p. 77-88.

16. Battagliani CLL. A randomized study on the effects of a prescribed exercise intervention on lean mass and fatigue changes in breast cancer patients during treatment. Greeley, CO: University of Northern Colorado; 2004. p. 2 I4

I7. Campbell A, Mutrie N, White F, et al. A pilot study of a supervised group exercise program as a rehabilitation treatment for women with breast cancer receiving adjuvant treatment. Eur J Oncol Nurs 2005;9:56-63.
I8. Courneya KS, Mackey JR, Bell GJ, et al. Randomized controlled trial of exercise training in postmenopausal breast cancer survivors: cardiopulmonary and quality of life outcomes. JClin Oncol 2003;21:166o-8.

I9. Crowley SA. The effect of a structured exercise program on fatigue, strength, endurance, physical self-efficacy, and functional wellness in women with early stage breast cancer. Ann Arbor, MI: University of Michigan; 2003. p. 127.

20. Drouin J. Aerobic exercise training effects on physical function, fatigue and mood, immune status, and oxidative stress in subjects undergoing radiation treatment for breast cancer. Detroit: Wayne State University; 2002. p. I-I42.

2I. Fairey AS, Courneya KS, Field CJ, et al. Effects of exercise training on fasting insulin, insulin resistance, insulin-like growth factors, and insulin-like growth factor binding proteins in postmenopausal breast cancer survivors: a randomized controlled trial. Cancer Epidemiol Biomarkers Prev 2003;12:72I-7.

22. Galantino ML, Capito L, Kane RJ, et al. Effects of Tai Chi and walking on fatigue and body mass index in women living with breast cancer: a pilot study. Rehabil Oncol 2003;21:17-22.

23. Kalda AL. The effect of upper body exercise on secondary lymphedema following breast cancer treatment. Vancouver: Faculty of Education, School of Human Kinetics, University of British Columbia; I999. p. 50.

24. MacVicar MG, Winningham ML. Promoting the functional capacity of cancer patients. Cancer Bull I986;38:235-9.

25. MacVicar MG, Winningham ML, Nickel JL. Effects of aerobic interval training on cancer patients' functional capacity. Nurs Res I989;38:348-5I.

26. McKenzie DC, Kalda AL. Effect of upper extremity exercise on secondary lymphedema in breast cancer patients: a pilot study. J Clin Oncol 2003;21:463-6.

27. Mock V, Burke MB, Sheehan P, et al. A nursing rehabilitation program for women with breast cancer receiving adjuvant chemotherapy. Oncol Nurs Forum I994; 21:899-907.

28. Mock V, Dow KH, Meares CJ, et al. Effects of exercise on fatigue, physical functioning, and emotional distress during radiation therapy for breast cancer. Oncol Nurs Forum 1997;24:99I-I000.

29. Mock V, Pickett M, Ropka ME, et al. Fatigue and quality of life outcomes of exercise during cancer treatment. Cancer Pract 200I;9:II9-27.

3o. Mock V, Frangakis C, Davidson NE, et al. Exercise manages fatigue during breast cancer treatment: a randomized controlled trial. Psychooncology 2005;I4(6):464-77.

3I. Mustian K. Breast cancer, Tai Chi Chuan, and self-esteem. Greensboro, NC: University of North Carolina; 2003. p. 217

32. Nieman DC, Cook VD, Henson DA, et al. Moderate exercise training and natural killer cell cytotoxic activity in breast cancer patients. Int J Sports Med I995;16:334-7.

33. Pickett M, Mock V, Ropka ME, et al. Adherence to moderate-intensity exercise during breast cancer therapy. Cancer Pract 2002;10:284-92.

34. Pinto BM, Frierson GM, Rabin C, et al. Home-based physical activity intervention for breast cancer patients. J Clin Oncol 2005;23:3577-87.

35. Schmitz KH, Ahmed RL, Hannan PJ, et al. Safety and efficacy of weight training in recent breast cancer survivors to alter body composition, insulin and insulin-like growth factor axis proteins. Cancer Epidemiol Biomarkers Prev 2005;I4:I672-80.

36. Schwartz AL, Winters K, Gallucci B. A randomized trial to examine effects of exercise on bone mineral density in premenopausal and postmenopausal women receiving chemotherapy for breast cancer. 2006. Unpublished manuscript.

37. Segal R, Evans W, Johnson D, et al. Structured exercise improves physical functioning in women with stages I and II breast cancer: results of a randomized controlled trial. J Clin Oncol 2001;19:657-65.

38. Segar ML, Katch VL, Roth RS, et al. The effect of aerobic exercise on self-esteem and depressive and anxiety symptoms among breast cancer survivors. Oncol Nurs Forum I998;25:107-I3.

39. Winningham ML, MacVicar MG. The effect of aerobic exercise on patient reports of nausea. Oncol Nurs Forum ig88;15:447-50.

40. Winningham ML, MacVicar MG, Bondoc M, et al. Effect of aerobic exercise on body weight and composition in patients with breast cancer on adjuvant chemotherapy. Oncol Nurs Forum I989;16:683-9.

4I. Ware JE, Snow KK, Kosinski MK, et al. SF-36 Health Survey manual and interpretation guide. Boston: Nimrod Press, 1993.

42. Cella D, Eton DT, Lai JS. Combining anchor and distribution based methods to derive minimal clinically important differences on the Functional Assessment of Cancer Therapy (FACT) anemia and fatigue scales. J Pain Symptom Manage 2002;24:547-61.

43. Myers J, Prakash M, Froelicher V, et al. Exercise capacity and mortality among men referred for exercise testing. N Engl J Med 2002;346:793-80I.

Correspondence to: Ms. Margaret L. McNeely, Faculty of Physical Education, University of Alberta, E-424 Van Vliet Center,

Edmonton $A B$ T6G 2H9; fax 780 492-8003;

mmcneely@ualberta.ca 